\title{
Adsorption-Desorption of Critical Metals from Simulated Acid Mine Drainage by Metakaolin Geopolymer
}

\author{
Esther Takaluoma ${ }^{1}$, Tatiana Samarina ${ }^{1}$ \\ ${ }^{1}$ Kajaani University of Applied Sciences \\ Kuntokatu 1, Kajaani, Finland \\ esther.takaluoma@kamk.fi
}

\begin{abstract}
Geopolymers are adsorbents of interest in the waste water treatment, as they can perform as cation exchanger for a wide variety of metals at circumneutral $\mathrm{pH}$. In here, the adsorption of copper and cobalt from a complex matrix, mimicked from real acid mine drainage was studied. Adsorbents at a dose from $5-40 \mathrm{~g} / \mathrm{L}$ was applied, and regenerated with sulfuric acid. The effect of regeneration on adsorption capacity is studied.
\end{abstract}

Keywords: geopolymer, adsorption, desorption, cobalt, copper

\section{Introduction}

Geopolymers have great potential for several applications within the framework of circular economy [1]. Thus, geopolymers or alkaline activated materials have recently found attention as adsorbents in the treatment of waste water, containing cationic contaminants [2][3]. Because geopolymers can be produced cheaply from a wide variety of industrial side streams, their selectivity can be tailored to the metal of concern. Nevertheless, water treatment by adsorption is intrinsically a non-zero waste technology, and therefore, it is crucial to regenerate adsorbents to make it economically and ecologically feasible, additionally recovery of critical metals such as copper and cobalt helps to provide new sources of raw materials. Recently, efforts have been made to improve the sustainability of the alkaline activation step, by utilizing side stream based activators [4].

\section{Materials and Methods}

All metal salts (Table 2), sulfuric acid (98\%), sodium hydroxide were purchased from VWR Chemicals and used as received. Sodium silicate - ZEOPOL 25 (42-46\%, molar ratio SiO2:Na2O is 2.4-2.6) was purchased from JV Huber. Kaolinite clay was obtained from Aquaminerals Finland Oy and calcined at $750{ }^{\circ} \mathrm{C}$ for three hours to obtain metakaolin (MK). The elemental composition of samples was measured semi-quantitatively with an X-ray fluorescence (XRF) spectrometer (PanAnalytical Minipal 4) with Omnian program.

\subsection{Metakaolin geopolymer preparation}

Metakaolin geopolymer (MKGP) was prepared according to literature procedure [5] as follows: Bulk geopolymers were prepared by mixing $100 \mathrm{~g}$ of solid metakaolin with alkaline activator in a solid to liquid ratio (S/L) of 1.1. Alkaline activator contained $8 \mathrm{M}$ sodium hydroxide and sodium silicate in a weight ratio of 1.1:1. The mixtures were allowed to consolidate at ambient temperature for three days, and the concrete-like monolith crushed with a jaw crasher, sieved, and washed with deionized water until $\mathrm{pH}$ 7.6. Fraction with particle size of $63-125 \mu \mathrm{m}$ was used for batch adsorption experiments. The composition of the geopolymer and its precursors is collected in Table 1 . The calcined MK consists mainly of silicon and aluminium, whereas potassium, titanium and iron are present as impurities. Sodium was introduced into the amorphous structure from activator solution. 
Table 1: Solid state composition of metakaolin geopolymer and its precoursers.

\begin{tabular}{|c|c|c|c|c|c|c|c|c|c|}
\hline Compound & $\mathrm{Na}_{2} \mathrm{O}$ & $\mathrm{Al}_{2} \mathrm{O}_{3}$ & $\mathrm{SiO}_{2}$ & $\mathrm{~K}_{2} \mathrm{O}$ & $\mathrm{Ti}$ & $\mathrm{V}$ & $\mathrm{Cr}$ & $\mathrm{Mn}$ & $\mathrm{Fe}_{2} \mathrm{O}_{3}$ \\
\hline kaolinite & & 53.661 & 65.573 & 2.735 & 0.051 & 0.001 & 0.003 & 0.014 & 1.590 \\
\hline metakaolin & & 42.522 & 66.274 & 6.624 & 0.701 & 0.026 & 0.022 & 0.005 & 2.960 \\
\hline MKGP & 5.281 & 20.963 & 46.408 & 4.209 & 0.434 & 0.006 & 0.015 & 0.002 & 2.405 \\
\hline
\end{tabular}

\subsection{Adsorption tests}

The adsorption experiments were performed using batch equilibration method. In the batch method, a portion of adsorbent $(1 \mathrm{~g} \mathrm{~L}-1-10 \mathrm{~g} \mathrm{~L}-1)$ was agitated with $200 \mathrm{ml}$ solution contained the metal salts dissolved in desalinated water $(0.055 \mu \mathrm{S} / \mathrm{cm})$ at $15 \mathrm{rpm}$ (overhead shaker) at room temperature for the desired time period to reach equilibrium. After filtration though paper filter (pore diameter $2.5 \mu \mathrm{m}$ ), analyses were performed with Perkin Elmer 500 Atomic adsorption spectrometer (ASS).

\subsection{Desorption tests}

To desorb the cationic metal from geopolymer adsorbent, batch desorption was utilized and the saturated powder was agitated with the appropriate amount of diluted $\mathrm{H}_{2} \mathrm{SO}_{4}$ solution. After two hours, the adsorbent was filtered off and the solution analysed by AAS.

\section{Discussion}

Adsorption tests were performed with modelled water based on real acid mine drainage of $\mathrm{pH} 2$ with the initial concentrations as seen in Table 2. Iron was removed by pre-treatment, e.g. by gradual rising of pH to 5 and filtering through celite. Adsorption tests were performed at $\mathrm{pH}$ 6. Care must be taken not to let $\mathrm{pH}$ rise over 8 to prevent rapid precipitation of metals as hydroxides, it is therefore crucial to wash the adsorbent until neutral. If alkaline activator remains in the geopolymer adsorbent, metal removal occurs as surface precipitation or by solution precipitation due to $\mathrm{pH}$ change.

Table 2: Concentration of contaminants in adsorption tests.

\begin{tabular}{|c|c|c|}
\hline Element & concentration $\mathbf{~ m g / L}$ & compound used \\
\hline $\mathrm{Co}$ & 10 & $\mathrm{CoCl}_{2}{ }^{*} 7 \mathrm{H}_{2} \mathrm{O}$ \\
\hline $\mathrm{Cu}$ & 110 & $\mathrm{CuCl}_{2}{ }_{2} \mathrm{H}_{2} \mathrm{O}$ \\
\hline $\mathrm{Mn}$ & 5 & $\mathrm{Mn}_{5} \mathrm{O}_{4}{ }^{*} \mathrm{H}_{2} \mathrm{O}$ \\
\hline $\mathrm{Fe}$ & 1500 & $\mathrm{FeSO}_{4}$ \\
\hline $\mathrm{K}$ & 12 & $\mathrm{KOH}$ \\
\hline $\mathrm{Na}$ & 20 & $\mathrm{Na}_{2} \mathrm{SO}_{4}$ \\
\hline
\end{tabular}

The dose dependency of the removal percent is depicted in Figure 1. Copper is preferentially adsorbed over cobalt, as the copper concentration, though ten times higher, reaches $100 \%$ faster than cobalt. A dose of $10 \mathrm{~g} / \mathrm{L}$ MKGP at initial concentrations of $110 \mathrm{mg} / \mathrm{l} \mathrm{Cu}$ and $10 \mathrm{mg} / \mathrm{L} \mathrm{Co}$, achieves a $91 \%$ copper removal and only a $31 \%$ cobalt removal efficiency, as seen in Figure 1. 


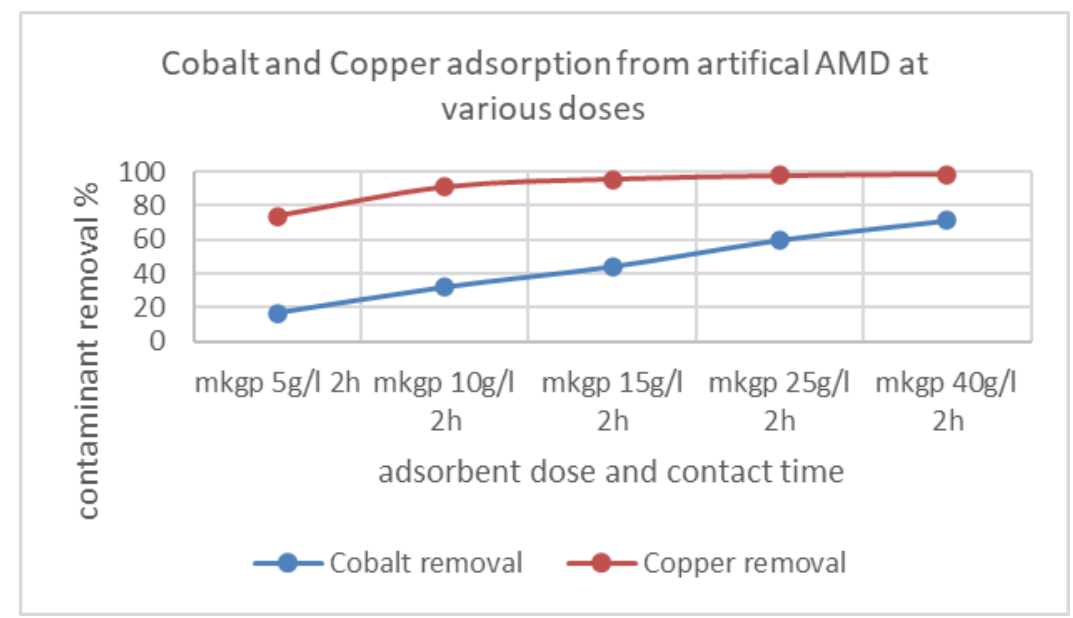

Figure 1: Dose-dependent study of cobalt and copper adsorption of MKGP at $2 \mathrm{~h}$ contact time

The adsorbents were cycled five times through adsorption-desorption cycle, utilizing $0.01 \mathrm{M} \mathrm{H}_{2} \mathrm{SO}_{4}$ and a 15 min contact time. The contact time was derived from initial time dependent studies of cobalt desorption from MKGP, where $15 \mathrm{~min}$ results in $91 \%$ desorption and $2 \mathrm{~h}$ contact time reaches over $96 \%$ cobalt desorption. The affect of continual desorption cycles on the adsorption capacity is depicted in Figure 2 and Figure 3. After the third loop, a rinsing with $5 \%$ vinegar facilitated the stabilization of adsorption capacity in the cycle four and five. In all cases, the adsorption capacity decreased substantially, most likely due to stronger interactions of the competing ions in the AMD with the adsorbent. However, higher concentrations of sulfuric acid quickly destroys the molecular, zeolitic channels within the geopolymer, and adsorption drops to zero. Rinsing with vinegar is possible at higher concentration, as vinegar is a softer, organic acid than sulfuric acid.

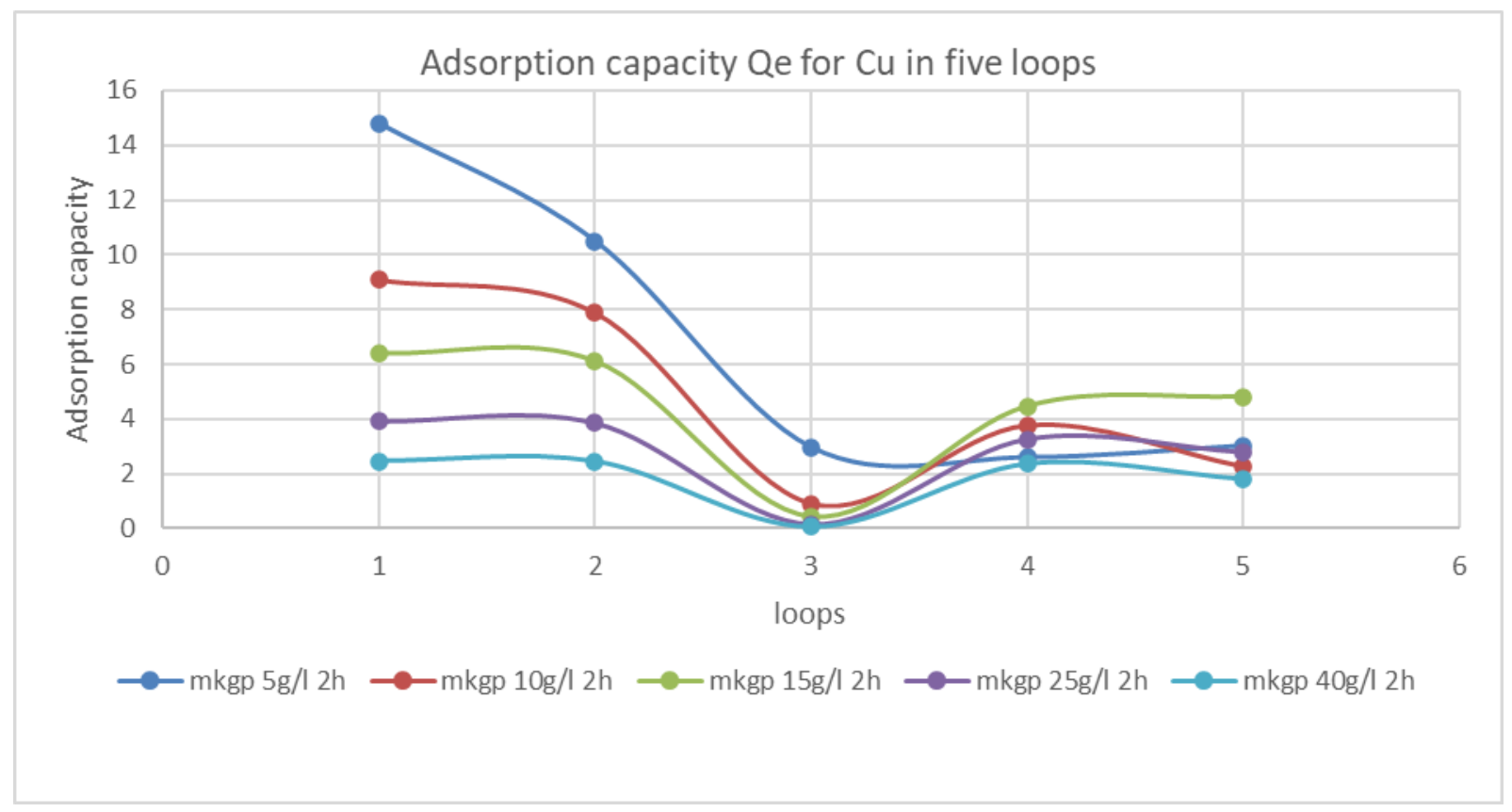

Figure 2: The effect of adsorbent cycling on adsorption capacity of copper on MK-GP at different doses. 


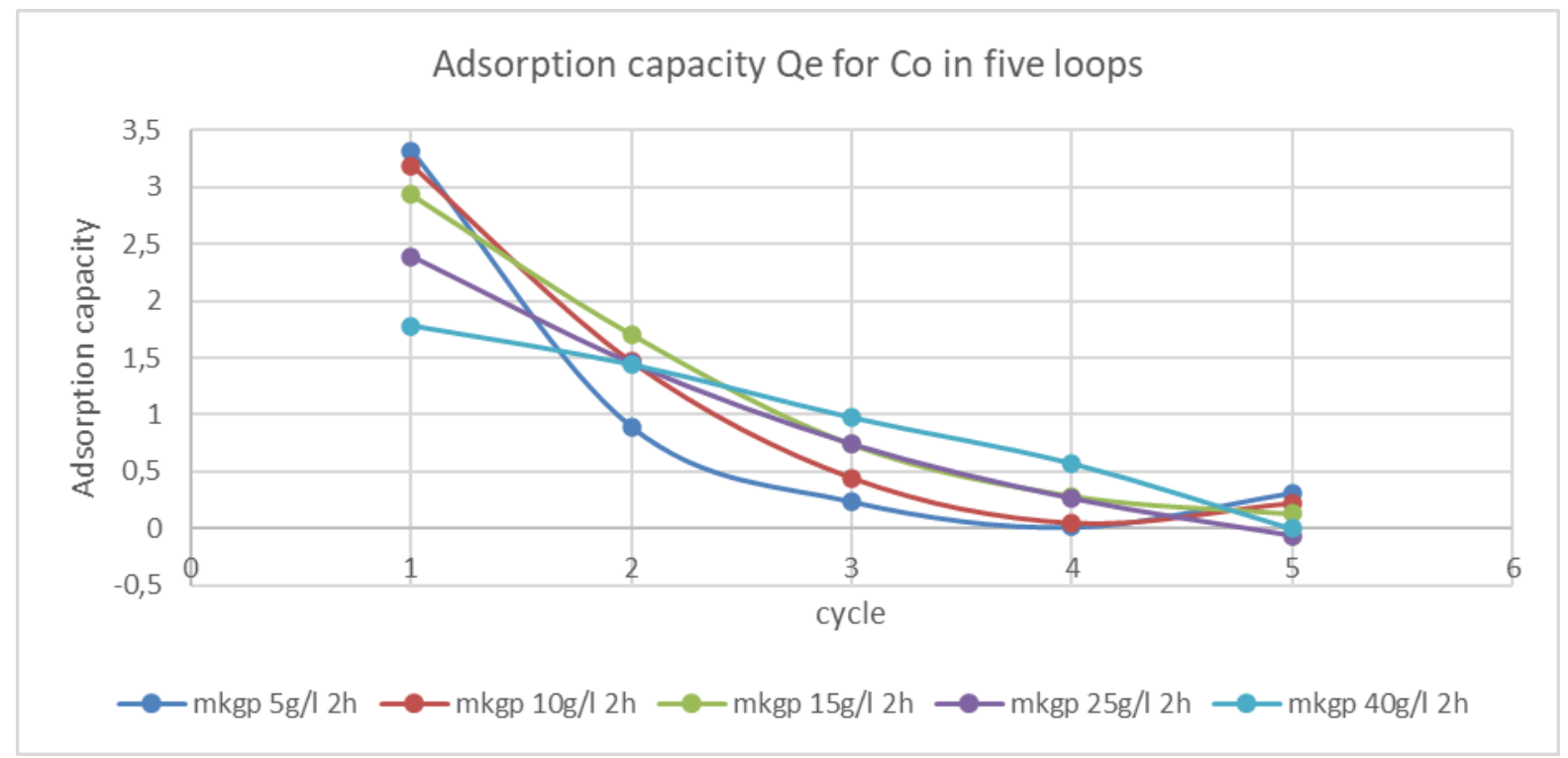

Figure 3: The effect of adsorbent cycling on adsorption capacity of cobalt on MK-GP at different doses.

\section{Conclusion}

This paper presents the cycling of sorption-desorption of copper and cobalt on metakaolin geopolymer adsorbent from complex ions matrix, based in real AMD. While adsorption capacity decreased significantly and more work needs to be done on regeneration of geopolymer adsorbents, these results spread light on desorption behaviour of critical materials.

\section{Acknowledgements}

This study was conducted as part of the WaterPro project (project number A74635), funded mainly by the European Regional Development Fund (EAKR, Keski-Pohjanmaan Liitto/Kainuun Liitto). The authors wish to thank Mrs. Marjukka Hyyryläinen for assisting with the laboratory work and analyses.

\section{References}

[1] R. Sefiu, B. Zhang, G. Rohiverth, T. Tiju, M. Yang, "Geopolymer for use in heavy metals adsorption, and advanced oxidative processes: A critical review," J. Clean. Prod., vol. 213, pp. 42-58, 2019.

[2] T.W. Cheng, M.L. Lee, M.S. Ko, T.H. Ueng, S.F. Yang, "The heavy metal adsorption characteristics on metakaolinbased geopolymer", Applied Clay Science, vol. 56, pp. 90-96, 2012.

[3] T. Samarina, E. Takaluoma, O. Laatikainen, "Geopolymers and Alkali-Activated Materials for Wastewater Treatment Applications and Valorization of Industrial Side Streams", 2021, DOI: 10.5772/intechopen.97141

[4] 1. Adesanya, P. Perumal, T. Luukkonen, J. Yliniemi, K. Ohenoja, P. Kinnunen, M. Illikainen, "Opportunities to improve sustainability of alkali-activated materials: A review of side-stream based activators", J. Clean. Prod., vol. 286, pp. 125558, 2021.

[4] L. Chen, Z. Wang, Y. Wang, J. Feng "Preparation and Properties of Alkali Activated Metakaolin-Based Geopolymer", Materials, 2016. 